\title{
Relation Between Serology of Meat Juice and Bacteriology of Tonsils and Feces for the Detection of Enteropathogenic Yersinia spp. in Pigs at Slaughter
}

\author{
Inge Van Damme, Gerty Vanantwerpen, Dirk Berkvens, ${ }^{2}$ and Lieven De Zutter ${ }^{1}$
}

\begin{abstract}
The association between positive serology and culture detection of Yersinia spp. in individual pigs was determined. Pieces of diaphragm from 370 pig carcasses were collected for serological analysis, and tonsils and feces of the same carcass were collected for bacteriological analysis. Detection of anti-Yersinia antibodies in meat juice samples was done using an indirect enzyme-linked immunosorbent assay (ELISA) based on Yops (Yersinia outer proteins). Tonsils and feces were tested for the presence of enteropathogenic Yersinia spp. by direct plating on cefsulodin-irgasan-novobiocin agar plates. Of the 370 meat juice samples, 241 (65.1\%) gave a positive serological reaction using a cutoff value of 20\%. Enteropathogenic Yersinia spp. (Yersinia enterocolitica serotype 0:3 and Yersinia pseudotuberculosis) were found in tonsils of 161 pigs and feces of 30 pigs. Recovery of enteropathogenic Yersinia from the tonsils was highly correlated with positive serotiters, whereas no correlation was found between serology and fecal excretion. Results demonstrated that serology has an acceptable sensitivity, but a relatively low specificity for the rapid detection of enteropathogenic Yersinia spp. in tonsils of pigs at slaughter.
\end{abstract}

\section{Introduction}

$\mathbf{E}$ NTEROPATHOGENIC YERSINIA SPP. (virulent strains of Yersinia enterocolitica and Yersinia pseudotuberculosis) are foodborne pathogens of the gastrointestinal tract, but have a strong tendency to extraintestinal spread under certain host conditions (Bottone, 1997; Long et al., 2010). All pathogenic Yersinia harbor a virulence plasmid (pYV), which encodes several yops (Yersinia outer proteins) that modulate the immune system and allow Yersinia to survive and replicate extracellularly within lymphoid tissues of the host (Heesemann et al., 2006).

$Y$. enterocolitica is mainly transmitted to humans through the consumption of contaminated pork (Fosse et al., 2008; Rosner et al., 2012). A high proportion of pigs at slaughter are infected with this pathogen, mainly bioserotype 4/O:3 (Bucher et al., 2008; Ortiz Martínez et al., 2009). Y. pseudotuberculosis is also found in slaughter pigs, though to a lesser extent than $Y$. enterocolitica (Ortiz Martínez et al., 2009). The pathogens are present in the oral cavity (particularly tonsils and tongue), lymph nodes, and the gastrointestinal tract. As pigs carry $Y$. enterocolitica without showing any symptoms, traditional meat inspection fails to detect infected pigs. During slaughter and dressing operations, enteropathogenic Yersinia spp. may spread to the rest of the carcass (Borch et al., 1996; Laukkanen et al., 2008, 2009).

To ensure an effective control of the main biological hazards on pork carcasses, preventive measures and controls should be applied both on-farm and at abattoir level. As carcass contamination with enteropathogenic Yersinia spp. depends on the contamination status of pigs delivered to the slaughterhouse (Laukkanen et al., 2008, 2009), incoming pig batches should be differentiated with respect to the risk they pose for public health (EFSA, 2011). Bacteriological monitoring of tonsils provides the best indication for the presence of Yersinia in slaughter pigs (Nesbakken et al., 2006), albeit time consuming and labor intensive (Fredriksson-Ahomaa and Korkeala, 2003). In contrast, serological screening of antibodies is a more convenient and faster alternative, given that the correlation between antibody titers and culture is high. Hence, the current study was conducted to assess the agreement between serological and bacteriological tests at animal level under field conditions. Accordingly, Yersinia antibodies in meat juice and the presence of enteropathogenic Yersinia spp. in tonsils and feces of pigs at slaughter were determined to estimate the relative sensitivity and specificity

\footnotetext{
${ }^{1}$ Department of Veterinary Public Health and Food Safety, Faculty of Veterinary Medicine, Ghent University, Merelbeke, Belgium.

${ }^{2}$ Unit of Veterinary Epidemiology, Department of Biomedical Sciences, Prince Leopold Institute of Tropical Medicine, Antwerp, Belgium.
} 
of the serology test for predicting the presence of Yersinia in slaughter pigs.

\section{Materials and Methods}

\section{Sample collection}

The tonsils, feces, and $10 \mathrm{~g}$ of diaphragm muscle of $370 \mathrm{pig}$ carcasses, originating from 194 farms (1-14 pigs per farm), were sampled during a 1-year period in 10 pig slaughterhouses in Belgium. Tonsils were excised aseptically after removal of the plug set and put in a sterile plastic bag. The whole intestinal tract was collected immediately after evisceration and the rectum was closed with threads, after which it was excised and put in a plastic bag. All samples were transported to the laboratory under refrigerated temperatures.

\section{Serological analysis}

Diaphragm muscles were frozen $\left(-20^{\circ} \mathrm{C}\right)$ immediately upon arrival for $24 \mathrm{~h}$ and then thawed at $4^{\circ} \mathrm{C}$ for $24 \mathrm{~h}$, after which the meat juice was collected and stored at $-20^{\circ} \mathrm{C}$ until analysis. Samples were examined for antibodies against pathogenic Yersinia using YOPSCREEN Pig enzyme-linked immunosorbent assay (ELISA) (Labor Diagnostik, Leipzig, Germany) according to the manufacturer's instructions. The presence of antibodies against Yops (Yersinia outer proteins) of plasmid-bearing Yersinia was determined by measuring the optical density (OD) at $450 \mathrm{~nm}$. Activity values (OD\%) were calculated based on OD values, relative to the mean OD values of positive and negative controls $\left(\mathrm{OD}_{\mathrm{pos}}\right.$ and $\mathrm{OD}_{\text {neg }}$, respectively).

$$
\text { Activity value }(O D \%)=\frac{O D_{\text {sample }}-O D_{\text {neg }}}{O D_{\text {pos }}-O D_{\text {neg }}}
$$

\section{Culture detection of enteropathogenic Yersinia spp.}

Tonsils and feces were examined for enteropathogenic Yersinia spp. by direct plating on cefsulodin-irgasan-novobiocin (CIN) (Bio-Rad, Nazareth, Belgium) agar plates (Van Damme et al., 2010). In short, $10 \mathrm{~g}$ of tissue was homogenized 1/10 (wt:wt) and $1 \mathrm{~mL}$ of homogenate was spread plated on two agar plates. Additionally, $100 \mu \mathrm{L}$ was inoculated on a CIN agar using a spiral plate machine (Eddie Jet, IUL Instruments, Barcelona, Spain). Human pathogenic $Y$. enterocolitica isolates were confirmed using polymerase chain reaction (PCR) assays targeting the ail, ystA, virF, $r f b C$, and per genes as described earlier (Van Damme et al.,
2013). Y. pseudotuberculosis isolates were identified using a PCR assay targeting the inv-gene (Nakajima et al., 1992).

\section{Statistical analyses}

Statistical analyses were performed using Stata/MP 12.1 (StataCorp, 2011). The relative sensitivity and specificity of the ELISA test using four different cutoff values $(10 \%, 20 \%$, $30 \%$, and $40 \%$ ) were estimated relative to culture of enteropathogenic Yersinia spp. from the tonsils and feces as criterion standard. For the remaining analyses, the proposed cutoff value of the manufacturer of $20 \%$ was used as cutoff value for a positive result. The proportion of positive pigs by each method was compared using a mixed-effects logistic regression analysis, including the individual pig and farm as random effects. The association between serology (positive/ negative) and culture results (presence/absence) was determined using a mixed-effects logistic regression, including the interaction between culture results from tonsils and feces. Farm was included as a random effect to account for clustering of pigs within a farm.

Based on bacteriological results, animals were classified in four different groups (group 1: tonsils and feces negative, group 2: tonsils negative and feces positive, group 3: tonsils and feces positive, and group 4: tonsils positive and feces negative). The seroprevalences within these groups were compared using a mixed-effects logistic regression including group as factor variable and farm as random effect. The activity values of seropositive pigs between groups were compared using a bootstrap median regression.

The association between lairage time (in hours) and bacteriological/serological results were analyzed using randomeffects logistic regressions, including slaughterhouse and farm as random effects whenever necessary. Only pigs for which the lairage time was known were used for the analysis $(n=321)$.

\section{Results}

The sensitivities and specificities of serology relative to culture examination of tonsils and feces are shown in Table 1. Compared to bacteriology of tonsils, the relative sensitivity and specificity of the ELISA test varied from $94.4 \%$ to $83.2 \%$ and from $41.6 \%$ to $69.9 \%$, depending on the applied cutoff value. The relative sensitivity and specificity were lower when serology was compared to fecal excretion as criterion standard. Using a cutoff value of $20 \%$, the sensitivities of the ELISA test were $91.9 \%$ and $63.3 \%$ compared to carriage of Yersinia in tonsils and excretion in feces as reference,

Table 1. Relative Sensitivities and Specificities of the Enzyme-Linked Immunosorbent Assay Test for Different Cutoff Values, Based on Bacteriological Results of Tonsils, Feces, and Both

\begin{tabular}{|c|c|c|c|c|c|c|}
\hline \multirow[b]{2}{*}{ Cutoff value } & \multicolumn{2}{|c|}{ Tonsils } & \multicolumn{2}{|c|}{ Feces } & \multicolumn{2}{|c|}{ Both $^{\mathrm{a}}$} \\
\hline & Sensitivity & Specificity & Sensitivity & Specificity & Sensitivity & Specificity \\
\hline $10 \%$ & 94.4 & 41.6 & 73.3 & 25.9 & 93.0 & 42.2 \\
\hline $20 \%$ & 91.9 & 55.5 & 63.3 & 34.7 & 89.5 & 55.8 \\
\hline $30 \%$ & 88.8 & 62.2 & 63.3 & 40.3 & 86.6 & 62.8 \\
\hline $40 \%$ & 83.2 & 69.9 & 56.7 & 47.1 & 80.1 & 69.9 \\
\hline
\end{tabular}

${ }^{a}$ Both: tonsils, feces, or at least one test is positive. 


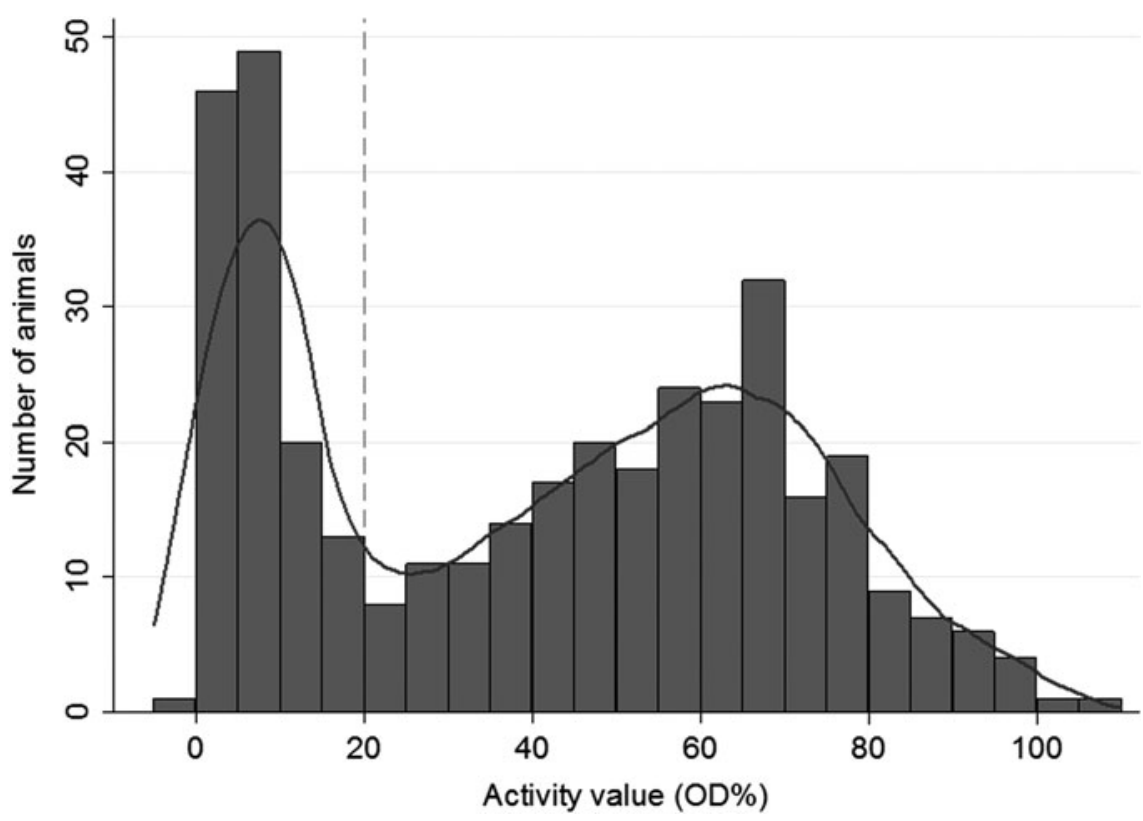

FIG. 1. Frequency distribution of the activity values (optical density [OD]\%) from meat-juice samples of 370 slaughter pigs. The solid line represents an Epanechnikov Kernel density distribution.

respectively. The relative specificities were $55.5 \%$ and $34.7 \%$, respectively.

The histogram of the activity values in Figure 1 shows a bimodal distribution of the serological data. Ninety-six samples $(25.9 \%)$ had an activity value below $10 \%$. Thirtythree samples $(8.9 \%)$ had an activity value between $10 \%$ and $20 \%$, and 19 samples $(5.1 \%$ ) between $20 \%$ and $30 \%$. In total, $65.1 \%$ of pigs were seropositive based on a cutoff value of $20 \%$. The average OD\% of seronegative and seropositive animals was $7.8 \pm 4.5(n=129)$ and 58.6 $18.7(n=241)$, respectively.

In total, enteropathogenic Yersinia spp. were recovered from 161 tonsils (43.5\%) and 30 fecal samples $(8.1 \%)$. Overall, 171 pigs (46.2\%) carried enteropathogenic Yersinia spp. in the tonsils and/or excreted them in the feces (Table 2). $Y$. enterocolitica serotype $0: 3$ was detected in the tonsils and feces of 159 and 30 pigs, respectively, whereas $Y$. pseudotuberculosis was recovered from 3 tonsils only, of which 1 pig had a concurrent $Y$. enterocolitica infection. The number

Table 2. Bacteriological and Serological Results of 370 Pigs at Slaughter

\begin{tabular}{lcccccccc}
\hline & \multicolumn{3}{c}{ Bacteriology } & & \multicolumn{3}{c}{ Serology (cutoff 20\%) } \\
\cline { 2 - 3 } Group & Tonsils & Feces & $\mathrm{n}^{\mathrm{a}}$ & & Positive & Negative & $\%^{\mathrm{b}}$ \\
\hline 1 & - & - & 199 & & 88 & 111 & 44.22 \\
2 & - & + & 10 & 5 & 5 & 50.00 \\
3 & $+{ }^{\mathrm{c}}$ & + & 20 & & 14 & 6 & 70.00 \\
4 & + & - & 141 & & 134 & 7 & 95.04 \\
Total & & & 370 & & 241 & & 129 & 65.14 \\
\hline
\end{tabular}

${ }^{\text {a }}$ Total number of samples within each group.

${ }^{\mathrm{b}}$ Seroprevalence within each group.

${ }^{\mathrm{c}}$ Positive for pathogenic Yersinia enterocolitica and/or Y. pseudotuberculosis (based on direct plating on cefsulodin-irgasannovobiocin agar; detection limit of 10 colony-forming units/g). of pigs showing serological evidence of infection (based on a cutoff of 20\%) was significantly higher than the number of pigs carrying enteropathogenic Yersinia in the tonsils $(p<0.001)$, both tests being significantly higher than the rate of pigs excreting enteropathogenic Yersinia spp. in the feces $(p<0.001)$.

From the 161 pigs that carried enteropathogenic yersiniae in the tonsils, $148(91.9 \%)$ tested serologically positive using a cutoff value of $20 \%$ (Table 2). Out of the 30 pigs that excreted the pathogens in the feces, $19(63.3 \%)$ were seropositive. Eighty-eight $(23.8 \%)$ of the tested pigs were serologically positive but showed no evidence of a current infection based on bacteriological results. A strong positive association was found between serology and the presence of enteropathogenic yersiniae in tonsils (adjusted odds ratio $[\mathrm{OR}]=64.37, p<0.001$ ), whereas no relation was found for fecal samples (adjusted $\mathrm{OR}=0.94, p=0.946$ ). Moreover, a negative interaction between tonsils and feces was found, which had borderline significance (adjusted $\mathrm{OR}=0.08, p=0.059$ ).

On the basis of bacteriological results from tonsils and feces, pigs were allocated into four groups (Table 2). The seroprevalence increased over the different groups, and was highest for pigs that carried Yersinia in the tonsils but did not excrete the pathogens in the feces in levels detectable by direct plating ( $>10$ colony-forming units $[\mathrm{CFU}] / \mathrm{g})(p=0.006)$. The distribution of the activity values within each bacteriological group is shown in Figure 2. In group 1 (tonsils and feces negative), the majority of pigs were seronegative, generally having an activity value below $10 \%$. The activity value of most seropositive pigs in this group was situated around 50\%. Contrarily, most pigs in group 4 (tonsils positive and feces negative) were seropositive, showing an activity value around $65 \%-70 \%$. The median activity values in seropositive pigs were significantly higher in groups 3 and 4 than in group $1(p<0.001)$.

The average holding time of the sampled pigs was $2.82 \mathrm{~h}$, with a standard error of $0.13 \mathrm{~h}$. The holding time of pigs that 


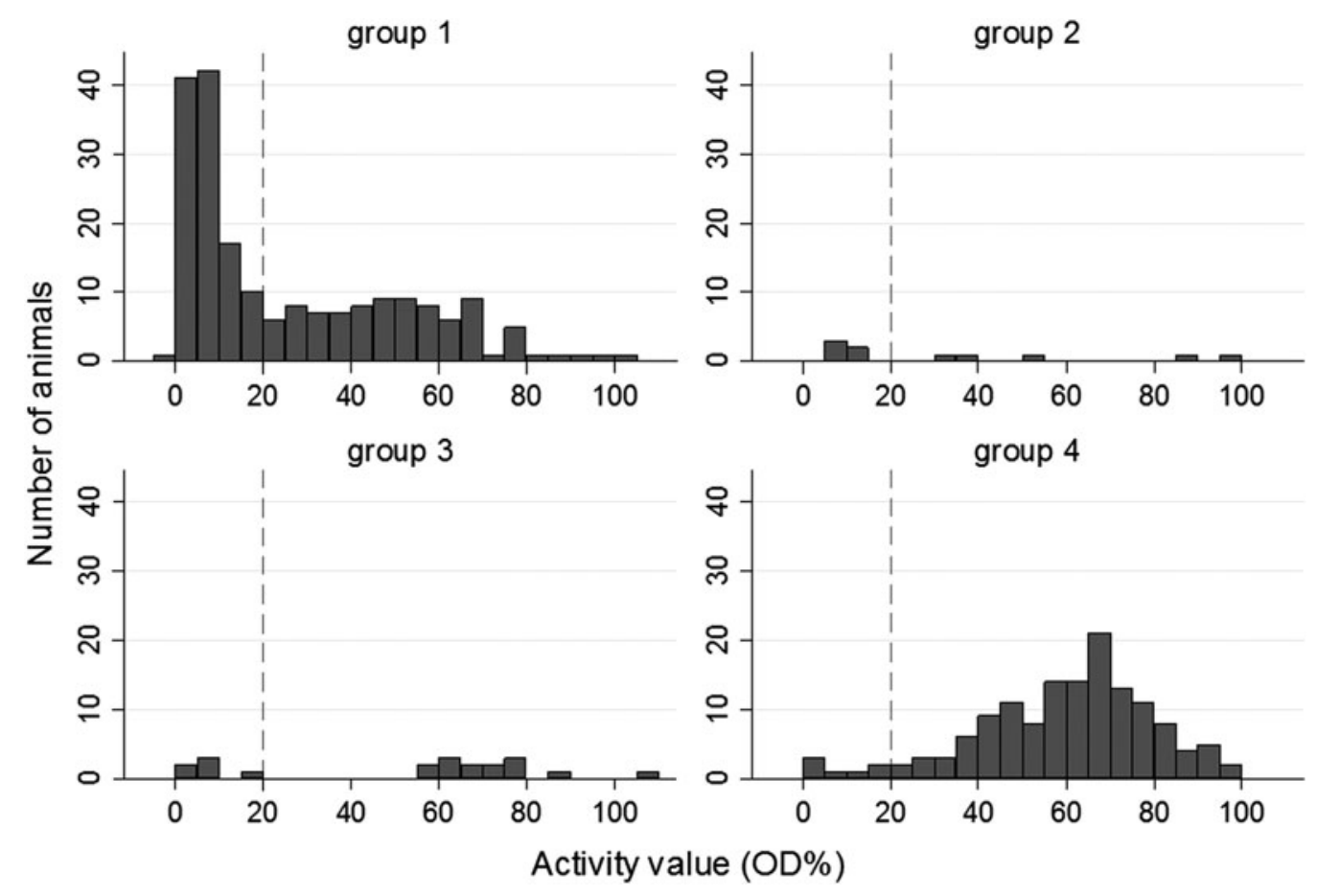

FIG. 2. Frequency distribution of activity values (optical density [OD]\%) of meat juice samples in different groups based on bacteriological results (tonsillar carriage and fecal excretion). Group 1: tonsils and feces negative; group 2: tonsils negative, feces positive; group 3: tonsils and feces positive; group 4: tonsils positive, feces negative.

excreted yersiniae in the feces $(3.95 \pm 0.57 \mathrm{~h})$ was significantly higher than that of pigs that did not excrete yersiniae $(2.73 \pm 0.13 \mathrm{~h})(p=0.015)$. No association was found between lairage time and tonsillar carriage $(p=0.311)$ nor between lairage time and the serological response $(p=0.908)$.

\section{Discussion}

This study evaluated the use of serology as an alternative to bacteriological testing to detect Yersinia infections in pigs. Antibodies were detected in $65 \%$ of pigs, while $43 \%$ carried enteropathogenic yersiniae in the tonsils and only a small proportion of animals excreted the pathogens in the feces (8\%). The ELISA test showed a high correlation and an acceptable sensitivity but limited specificity when compared to bacteriological culture of tonsils as reference.

In contrast to culture examination, a serological reaction is a historical measure of infection, which inherently limits the specificity of serology when compared to bacteriology. Although some animals may test negative for enteropathogenic Yersinia in the tonsils and feces after a certain period, the serological reaction remains positive for at least 70 days after infection (Nielsen et al., 1996). The activity values of seropositive pigs were lower in culture-negative animals than in pigs that carried yersiniae in the tonsils, which may represent the decrease in antibodies after elimination of infection. Nevertheless, besides the biological differences between serology and bacteriology, a high association was found between seropositivity and isolation of Yersinia from the tonsils, whereas no association was found between serology and fecal Yersinia excretion. Similarly, von Altrock et al. (2006) analyzed blood and feces from 900 pigs in 30 fattening-pig herds shortly before slaughter and no correlation was found between serological and culture results. Pigs can carry the pathogen in tonsils for several months, whereas fecal excretion declines more rapidly (Nielsen et al., 1996; Virtanen et al., 2012). However, the time period after experimental infection in which Yersinia are detectable in the feces is influenced by the isolation method used, and possibly results from a decline in numbers over time (Nielsen et al., 1996). As only direct plating was used in the present study, only animals excreting higher numbers $(>10 \mathrm{CFU} / \mathrm{g})$ were detected, which may indicate that they were relatively more recently infected. Recently infected animals (less than 2-3 weeks before slaughter) are less likely to have seroconverted, which might partly explain the low relative sensitivity of the serological test to detect excretion of enteropathogenic Yersinia spp. in the feces in the present study.

Pathogenic $Y$. enterocolitica can be recovered from tonsils and intestines within hours after oral inoculation of pigs (Thibodeau et al., 1999), whereas seroconversion is observed after 12-19 days of experimental infection (Nielsen et al., 1996). Similarly, Nesbakken et al. (2006) followed pigs on two naturally infected Yersinia-positive farms and observed that contamination of tonsils and feces started around the same time, whereas seroconversion occurred about 2 weeks later. In the present study, only a small part of the tested pigs $(18 / 370,5 \%)$ were serologically negative while they carried and/or excreted pathogenic Yersinia in the tonsils and feces, which may indicate a recent infection. Initial infection may have taken place on-farm during the final stage of the fattening period, but also during transport or lairage at the abattoir.

The average lairage time for all pigs was almost $3 \mathrm{~h}$, which is sufficiently long for Yersinia to colonize the tonsils and intestines under experimental conditions (Thibodeau et al., 
1999). Nevertheless, only for fecal excretion a significant association was found with longer lairage times. For every increase in holding time with $1 \mathrm{~h}$, the odds of finding enteropathogenic Yersinia spp. in the feces increased with a factor 1.20. This association may indicate that the risk of infection (and fecal excretion) increases with longer lairage times or that fecal excretion increases with increasing holding times (e.g., due to stress).

Several factors may influence serological results, such as the applied antigen, sample type, and cutoff value. The present study used muscle fluid to detect Yersinia antibodies in pigs at slaughter as it is a faster and easier alternative compared to serum. The ELISA test contains Yop antigens, so that both human pathogenic $Y$. enterocolitica and $Y$. pseudotuberculosis antibodies are detected. On the other hand, ELISA assays using specific antigens against $Y$. enterocolitica serotype O:3 (Nielsen et al., 1996) may detect antibodies against the most common pathogenic serotype in pigs, but not the more rare $Y$. enterocolitica serotypes and $Y$. pseudotuberculosis. Nevertheless, as in most European countries, $Y$. enterocolitica serotype O:3 predominates in pigs (Bucher et al., 2008; Ortiz Martínez et al., 2009; Van Damme et al., 2010), this may have a small impact on the final outcome.

The activity values showed a bimodal distribution with modes at about $5 \%$ and $65 \%$, which may represent the seronegative and seropositive population, respectively. Accordingly, this distribution would suggest that the cutoff value for the ELISA test should be between $20 \%$ and $30 \%$ and indicates that the proposed cutoff value of $20 \%$ of the manufacturer corresponds with the field data.

Besides test-related factors, biological aspects such as age of the pigs and prevalence differences (e.g., between regions and farms) may also affect the serological outcome. Nevertheless, despite many influencing factors, the proportion of slaughter pigs with Yersinia antibodies is usually very similar among different studies, as the seroprevalence found here $(65 \%)$ agrees with the $66 \%$ in Canada (Thibodeau et al., 2001 ) and $64 \%$ found in fattening pigs just before or during slaughter in Germany (von Altrock et al., 2011).

\section{Conclusions}

Serology of meat juice of pigs at slaughter correlates well with the carriage of pathogenic Yersinia in tonsils, whereas serological findings show no relation with Yersinia excretion in the feces. The meat juice ELISA can thus be used as an indicator for Yersinia carriage at the individual level, though the low epidemiologic specificity may result in many falsepositive reactions.

\section{Acknowledgments}

The authors thank Annelies Wachtelaer and Anne-Mie Terryn for the technical assistance. The slaughterhouses are kindly acknowledged for their cooperation in sampling. This study was funded by a doctoral grant from the Special Research Fund (BOF, Bijzonder Onderzoeksfonds, BOF09/24J/ 064) of Ghent University, Belgium.

\section{Disclosure Statement}

No competing financial interests exist.

\section{References}

Borch E, Nesbakken T, Christensen H. Hazard identification in swine slaughter with respect to foodborne bacteria. Int J Food Microbiol 1996;30:9-25.

Bottone EJ. Yersinia enterocolitica: The charisma continues. Clin Microbiol Rev 1997;10:257-276.

Bucher M, Meyer C, Grotzbach B, Wacheck S, Stolle A, Fredriksson-Ahomaa M. Epidemiological data on pathogenic Yersinia enterocolitica in Southern Germany during 20002006. Foodborne Pathog Dis 2008;5:273-280.

[EFSA] European Food Safety Authority. Technical specifications for harmonised national surveys of Yersinia enterocolitica in slaughter pigs on request of EFSA. EFSA J 2009;7:1374.

EFSA. Scientific opinion on the public health hazards to be covered by inspection of meat (swine). EFSA J 2011;9:2351.

Fosse J, Seegers H, Magras C. Foodborne zoonoses due to meat: A quantitative approach for a comparative risk assessment applied to pig slaughtering in Europe. Vet Res 2008;39:1.

Fredriksson-Ahomaa M, Korkeala H. Low occurrence of pathogenic Yersinia enterocolitica in clinical, food, and environmental samples: A methodological problem. Clin Microbiol Rev 2003;16:220-229.

Heesemann J, Sing A, Trulzsch K. Yersinia's stratagem: Targeting innate and adaptive immune defense. Curr Opin Microbiol 2006;9:55-61.

Laukkanen R, Martinez PO, Siekkinen KM, Ranta J, Maijala R, Korkeala H. Transmission of Yersinia pseudotuberculosis in the pork production chain from farm to slaughterhouse. Appl Environ Microb 2008;74:5444-5450.

Laukkanen R, Martinez PO, Siekkinen KM, Ranta J, Maijala R, Korkeala H. Contamination of carcasses with human pathogenic Yersinia enterocolitica 4/O:3 originates from pigs infected on farms. Foodborne Pathog Dis 2009;6:681-688.

Long C, Jones TF, Vugia DJ, Scheftel J, Strockbine N, Ryan P, Shiferaw B, Tauxe RV, Gould LH. Yersinia pseudotuberculosis and Y. enterocolitica infections, FoodNet, 1996-2007. Emerg Infect Dis 2010;16:566-567.

Nakajima H, Inoue M, Mori T, Itoh K, Arakawa E, Watanabe H. Detection and identification of Yersinia pseudotuberculosis and pathogenic Yersinia enterocolitica by an improved polymerase chain reaction method. J Clin Microbiol 1992;30:2484-2486.

Nesbakken T, Iversen T, Eckner K, Lium B. Testing of pathogenic Yersinia enterocolitica in pig herds based on the natural dynamic of infection. Int J Food Microbiol 2006;111: 99-104.

Nielsen B, Heisel C, Wingstrand A. Time course of the serological response to Yersinia enterocolitica O:3 in experimentally infected pigs. Vet Microbiol 1996;48:293-303.

Ortiz Martínez P, Fredriksson-Ahomaa M, Sokolova Y, Roasto M, Berzins A, Korkeala H. Prevalence of enteropathogenic Yersinia in Estonian, Latvian, and Russian (Leningrad region) pigs. Foodborne Pathog Dis 2009;6:719-724.

Rosner BM, Stark K, Höhle M, Werber D. Risk factors for sporadic Yersinia enterocolitica infections, Germany 20092010. Epidemiol Infect 2012;140:1738-1747.

StataCorp. Stata: Release 12. Statistical Software. College Station, TX: StataCorp LP, 2011.

Thibodeau V, Frost EH, Chenier S, Quessy S. Presence of Yersinia enterocolitica in tissues of orally-inoculated pigs and the tonsils and feces of pigs at slaughter. Can J Vet Res 1999; 63:96-100.

Thibodeau V, Frost EH, Quessy S. Development of an ELISA procedure to detect swine carriers of pathogenic Yersinia enterocolitica. Vet Microbiol 2001;82:249-259. 
Van Damme I, Berkvens D, Botteldoorn N, Dierick K, Wits J, Pochet B, De Zutter L. Evaluation of the ISO 10273:2003 method for the isolation of human pathogenic Yersinia enterocolitica from pig carcasses and minced meat. Food Microbiol 2013;36:170-175.

Van Damme I, Habib I, De Zutter L. Yersinia enterocolitica in slaughter pig tonsils: Enumeration and detection by enrichment versus direct plating culture. Food Microbiol 2010;27: $158-161$.

Virtanen S, Salonen L, Laukkanen-Ninios R, FredrikssonAhomaa M, Korkeala H. Piglets are a source of pathogenic Yersinia enterocolitica on fattening-pig farms. Appl Environ Microbiol 2012;78:3000-3003.

von Altrock A, Louis AL, Rosler U, Beyerbach M, Kreienbrock L, Waldmann KH. Study on the bacteriological and serological prevalence of Campylobacter spp. and Yersinia enterocolitica in fattening pig herds in Lower Saxony. Berliner Munch Tierarzt Wochenschr 2006;119:391-399.

von Altrock A, Roesler U, Waldmann KH. Herd factors associated with the serological Yersinia prevalence in fattening pig herds. Foodborne Pathog Dis 2011;8:1249-1255.

Address correspondence to: Inge Van Damme, DVM, PhD Department of Veterinary Public Health and Food Safety

Faculty of Veterinary Medicine, Ghent University

B-9820 Merelbeke, Belgium

E-mail: inge.vandamme@ugent.be 